\title{
Microalgal symbiosis in biotechnology
}

\author{
Carla A. Santos • Alberto Reis
}

Received: 31 January 2014 /Revised: 7 April 2014 / Accepted: 8 April 2014 / Published online: 10 May 2014

(C) Springer-Verlag Berlin Heidelberg 2014

\begin{abstract}
This review provides an analysis of recent published work on interactions between microorganisms, especially the ones involving mainly nutrient exchanges and at least with one microalga species. Examples of microbial partners are given, with a remark to the potential application of cultures of an autotroph and a heterotroph, which grow simultaneously, taking advantage of the complementary metabolisms. These are particularly interesting, either due to economic or sustainable aspects, and some applications have already reached the commercial stage of development. The added advantages of these symbiotic cultures are biomass, lipid, and other products productivity enhancement a better utilization of resources and the reduction or even elimination of process residues (including carbon dioxide and other greenhouse gases) to conduct an increasingly greener biotechnology. Among the several symbiotic partners referred, the microalgae and yeast cultures are the most used. The interaction between these two microorganisms shows how to enhance the lipid production for biodiesel purposes compared with separated (stand-alone) cultures.
\end{abstract}

Keywords Symbiosis · Mutualism · Commensalism · Microalgae $\cdot$ Autotrophy $\cdot$ Heterotrophy

\section{Introduction}

There are symbioses in nature since the beginning of life on Earth and mankind grasped the opportunity to apply these phenomena in biotechnology processes. Symbiosis, which simply means living together, is a relationship wide spread

C. A. Santos $\cdot$ A. Reis $(\bowtie)$

LNEG, Unidade de Bioenergia, Estrada do Paço do Lumiar, 22, ed. F,

1649-038 Lisbon, Portugal

e-mail: alberto.reis@lneg.pt in nature that defines an association between dissimilar organisms, divided in types according to the advantages that the partners obtain from it. Mutual symbiosis occurs when both partners get advantages from the association. Commensalism occurs when one partner gains an advantage but the other is not damaged in the association. When one of the partners is damaged, the association is called parasitism (Schlegel 1986; Black 1996; Norton 1985). This review focuses only on the positive interaction types, such as mutualism and commensalism.

There are examples of symbioses in all kingdoms of living beings and the most widespread reason for that is nutrient supply (Honegger 2008; Ahmadjian and Paracer 2000). The same interactions can be found among fungi, bacteria, microalgae, and cyanobacteria, which are the main target of biotechnology processes.

A well-known example from nature is that of lichens, commonly found on rocks and trees as crusts, which are associations of fungi with photosynthetic algae or cyanobacteria (Prescott et al. 1999; Oksanen 2006). The fungus and alga are so closely associated that they make up a unitary vegetative body growing attached to rocks, tree trunks, and other unlikely habitats. The fungus obtains its organic nutrients from an alga that fixes $\mathrm{CO}_{2}$ by photosynthesis. In turn, the fungus protects the alga and supplies it with water and minerals obtained from the atmosphere. These partners in lichen symbiosis can be separated and will grow as individual fungi or algae. It is also possible to construct lichens (Schlegel 1986).

Some of the most interesting characteristics of lichens are the production of secondary metabolites, tolerance to extreme environments, and sensitivity to pollution. Industrially important secondary metabolites isolated from lichens are unique to this symbiosis and have application in medicine, perfume industry, brewing, dying industry, and food industry (Oksanen 2006; Müller 2001). Some authors report that more 